\title{
Migraine and risk of incident diabetes in women: prospective study
}

\author{
Rebecca C. Burch, MD*, Pamela M. Rist, ScD*, Anke C. Winter, MD, MSc, Julie E. Buring, \\ ScD, Aruna D. Pradhan, MD, MSc, Elizabeth W. Loder, MD, MPH, and Tobias Kurth, MD, \\ ScD \\ Division of Headache and Pain (R.C.B, E.W.L.), Department of Neurology and the Division of \\ Preventive Medicine (P.M.R., A.C.W., J.E.B., A.D.P., T.K.), Department of Medicine, Brigham and \\ Women's Hospital, Harvard Medical School, Boston, MA; the Department of Epidemiology \\ (P.M.R., J.E.B., T.K.), Harvard School of Public Health, Boston, MA; INSERM Unit 708- \\ Neuroepidemiology (T.K.), Bordeaux, France, and University of Bordeaux (T.K.), Bordeaux, \\ France
}

\begin{abstract}
Background-Previous cross-sectional studies evaluating the relationship between diabetes prevalence and migraine status have found conflicting results. We examined the relationship between migraine and incident type 2 diabetes (T2D) in a cohort of adult women.
\end{abstract}

\begin{abstract}
Methods-Prospective cohort study conducted among participants in the Women's Health Study who provided information on migraine and did not have diabetes at baseline. Our four exposure groups were migraine with aura, migraine without aura, past history of migraine and no history of migraine. Cox proportional hazards models were used to determine the hazard ratio for incident T2D.
\end{abstract}

Results-Among the 38,620 women included in this study, $5062(13.1 \%)$ women had migraine, of whom 2014 (39.8\%) reported migraine with aura, and 2,087 (5.4\%) women had a past history of migraine. During a mean of 14.6 years of follow-up, there were 3,032 cases of incident T2D. After adjustment for confounders, the hazard ratio (95\% confidence interval) for developing diabetes was $1.06(0.91-1.24)$ for women with migraine with aura, $1.01(0.89-1.16)$ for women

\footnotetext{
Corresponding Author: Tobias Kurth, MD, ScD, INSERM U708-Neuroepidemiology, Université Bordeaux Segalen, 146 rue Léo Saignat, Bat. Isped, case 11, 33076 Bordeaux cedex, France, Tel: +33 5575713 79, tobias.kurth@univ-bordeaux.fr. Contributed equally

\section{Conflicts of Interest}

The authors report no conflicts of interest with regard to the specific matter of this study but list full disclosures for the past two years: Dr. Burch is supported by a fellowship from the American Headache Society.

Dr. Rist has received funding from a training grant from the National Institute of Aging (AG00158), from the Rose Traveling Fellowship Program in Chronic Disease Epidemiology and Biostatistics from the Harvard School of Public Health, and from a travel fund from the Department of Epidemiology at the Harvard School of Public Health.

Dr. Winter has received an international postdoctoral fellowship of the American Association of University Women and a research fellowship of the German Research Foundation (DFG).

Dr. Buring has received investigator-initiated research funding and support as Principal Investigator from the US National Institutes of Health and research support for pills and/or packaging from Bayer Heath Care and the Natural Source Vitamin E Association. Dr. Loder receives salary support from the British Medical Journal for services as a clinical editor.

Dr. Pradhan receives funding from the US National Institutes of Health and has received investigator-initiated research support from Sanofi-Aventis. She has received honoraria from Roche Diagnostics for educational lectures.

Dr. Kurth has received investigator-initiated research funding from the National Institutes of Health, the French National Research Agency (ANR, Agence Nationale pour la Recherche), Merck, the Migraine Research Foundation and the Parkinson's Disease Research Foundation. He is a consultant to World Health Information Science Consultants, LLC and he has received honoraria from the American Academy of Neurology and Merck for educational lectures, from MAP Pharmaceutical for contributing to a scientific advisory panel, and from the British Medical Journal for editorial services.
} 
with migraine without aura, $1.13(0.98-1.30)$ for women with a past history of migraine compared to women with no history of migraine.

Conclusion-Results of this prospective study in women do not support an association between migraine and incident T2D.

\section{Keywords}

migraine; diabetes; epidemiology

\section{Introduction}

Migraine is a primary headache disorder affecting approximately $6 \%$ of men and $18 \%$ of women (1). In about one third of patients, migraine may be associated with transient neurologic symptoms, usually visual, known as migraine aura (2). Prior studies have shown that migraine with aura is associated with an increased risk of ischemic stroke and cardiovascular disease (3-8). Migraine, particularly migraine with aura, is also associated with increased prevalence of cardiovascular risk factors $(9,10)$ such as hypertension and smoking, as well as vascular biomarkers $(11,12)$, particularly increased cholesterol levels.

Metabolic syndrome has been proposed as one possible mechanism to explain the relationship between migraine, obesity, and increased cardiovascular disease $(13,14)$. One component of the metabolic syndrome is insulin resistance. Several studies have shown impaired insulin sensitivity in migraineurs, including elevated glucose and insulin levels in both the fasting state and post-load during glucose tolerance testing $(9,15-17)$. This has led to speculation that migraineurs may also be at increased risk for diabetes. Results from the population-based American Migraine Prevalence and Prevention Study (AMPP) showed that subjects with migraine in the last year were more likely than headache-free controls to report a diagnosis of T2D (type 2 diabetes) (3). However, this cross-sectional analysis could not evaluate the association between migraine and risk of future T2D. Therefore, we aimed to investigate the association between migraine status and incident T2D using data from the Women's Health Study, a large ongoing prospective cohort study.

\section{Methods}

The Women's Health Study (WHS) was a randomized double-blinded placebo controlled trial designed to examine the effects of low-dose aspirin and vitamin $\mathrm{E}$ in the primary prevention of cardiovascular disease and cancer. The design and methods of the trial have been previously described (18). Briefly, 39,876 US female health professionals aged $\geq 45$ years without a history of cardiovascular disease, cancer or other major illnesses were randomized to receive active aspirin, active vitamin $\mathrm{E}$, both active agents, or both placebos at baseline (1993-1996). After the end of the clinical trial in March 2004 (19, 20), the women continue to be followed on an observational basis. On the baseline questionnaire, the women were asked about personal characteristics, medical history (including migraine and diabetes) and other health information. Yearly follow-up questionnaires asked about study compliance and medical events, including new diagnosis of diabetes. All participants provided informed consent and the institutional review board at Brigham and Women's Hospital approved the WHS.

\section{Migraine Assessment}

On the baseline questionnaire, women were asked: "Have you ever had migraine headache?" and "In the past year, have you had migraine headaches?" If a woman reported migraine headaches within the past year, she was asked about characteristics of her migraines 
including the presence of aura or any indication that a migraine is coming. Women who reported ever experiencing migraine headache were considered to have "any history of migraine". For stratified analyses, we divided the women into four mutually exclusive categories: (1) "no migraine history; (2) "active migraine with aura," which included those who reported migraine within the past year and the presence of aura or any indication that a migraine is coming; (3) "active migraine without aura"; and (4) "history of migraine," which included women who reported ever having migraines but did not experience a migraine in the past year.

Previous validation studies in two subsamples of the WHS have shown that among women with active migraine, $83.5 \%$ fulfilled all but one International Classification of Headache Disorders-I (ICHD-I) criteria for migraine (code 1.7, migrainous disorder) and $46.6 \%$ fulfilled all ICHD-I criteria for "migraine without aura" (code 1.1, migraine without aura) (4). Additionally, $87.7 \%$ of women with self-reported active migraine have migraine without aura $(71.5 \%)$ or probable migraine without aura $(16.2 \%)$ according to the ICHD-II criteria (21).

\section{Diabetes Assessment}

On the baseline questionnaire, women were asked: "Have you ever had diabetes mellitus, diagnosed prior to age 30?" and "Have you ever had diabetes mellitus, diagnosed at 30 or older?" Annually thereafter, the women were asked if they had been diagnosed with diabetes since completing the previous questionnaire. Self-reported cases were then confirmed by physician-administered telephone interviews using the American Diabetes Association diagnostic criteria (22) or a self-administered supplemental questionnaire. In a validation study, both interview-based and supplemental questionnaire-based confirmation yielded positive predictive values $>90 \%$ in comparison to medical record review (23). In particular, the positive predictive value of the supplemental questionnaire was $99 \%$ (95\% CI 97$100 \%)$. Overall, in 95\% of all-self-reported T2D events, sufficient information for confirmation or disconfirmation of the endpoint was obtained, and only confirmed cases were used in this analysis. Because the vast majority of diabetes diagnosed at age $>45$ years is of the type 2 variant, incident diabetes in the WHS is considered to be type 2 diabetes.

\section{Analysis}

Of the 39,876 women eligible for this study, we excluded the 119 women who did not report migraine status at baseline, leaving 39,757 women for this analysis. At baseline, 1137 women reported a history of diabetes mellitus. We used age- and multivariable-adjusted logistic regression to determine the cross-sectional association between migraine and diabetes at baseline using women without a history of migraine as the reference group. We then further stratified our results by migraine subtype (migraine with aura, migraine without aura and past history of migraine).

For our longitudinal analyses, we excluded the 1137 women with a baseline history of diabetes. The baseline characteristics of the women without diabetes can be seen in Table 1 . We used age- and multivariable -adjusted Cox proportional hazards models to determine the hazard ratio (HR) for incident diabetes using women without a history of migraine as the reference group. We tested the assumption of proportional hazards by including an interaction term between the $\log$ transformation of time in the study and migraine status. No significant violation was found.

The cross-sectional and longitudinal multivariable-adjusted models adjusted for the following potential confounders: age (continuous), body mass index $\left(<18 \mathrm{~kg} / \mathrm{m}^{2}, 18\right.$ to $<25$ $\mathrm{kg} / \mathrm{m}^{2}, 25$ to $<30 \mathrm{~kg} / \mathrm{m}^{2}, 230 \mathrm{~kg} / \mathrm{m}^{2}$ ), exercise (rarely/never, $<1$ time per week, 1 to 3 times 
per week, $\geq 4$ times per week), ever used postmenopausal hormones of any type (never, past, current), postmenopausal status (premenopausal, postmenopausal, uncertain), alcohol consumption (rarely/never, 1 to 3 drinks per month, 1 to 6 drinks per week, $\geq 1$ drink per day), history of hypertension $\geq 140 / 90 \mathrm{mmHg}$ (yes/no), baseline treatment with medication for high blood pressure (yes/no), history of high cholesterol $\geq 240 \mathrm{mg} / \mathrm{dL}$ (yes/no), baseline treatment with cholesterol-lowering medication (yes/no), smoking status (never, past, smoke $<15$ cigarettes per day, smoke $\geq 15$ cigarettes per day), systolic blood pressure $(10 \mathrm{mmHg}$ increments), family history of diabetes (yes/no), and ever used oral contraceptives $\geq 2$ months (yes/no). We additionally adjusted for randomized treatment assignment to aspirin and vitamin $\mathrm{E}$ (yes/no).

In secondary analyses, we tested for effect modification of the association between migraine and diabetes by randomized treatment assignment to aspirin, currently taking non-steroidal anti-inflammatory drugs (NSAIDs) more than 1 day per week, history of hypertension $\geq 140 / 90 \mathrm{mmHg}$, baseline treatment with medication for high blood pressure, obesity, and age ( $<54.6$ years or $\geq 54.6$ years, the mean age of the cohort). To test for effect modification, we included an interaction term between migraine and each potential effect modifier in separate multivariable adjusted models. We also examined whether the risk of diabetes varied based on the frequency of active migraine by running models stratified by migraine frequency ( $<6$ times per years, every other month, monthly, or weekly).

More than 100 women were missing information on systolic blood pressure category and were assigned to a separate category. Less than 100 women were missing information on all other covariates and were assigned to the reference category (ie, lowest exposure), past user category (smoking) or unclear exposure category (postmenopausal hormone use).

All statistical analyses were performed in SAS 9.1. All p-values were 2-tailed and $p<0.05$ was considered statistically significant.

\section{Results}

Of the 39,757 women who reported information on migraine and diabetes status at baseline, 5,174 women (13.0\%) reported active migraine in the past year, of whom 2,059 (39.8\%) reported migraine with aura, and 2,156 women (5.4\%) reported a previous history of migraine. There were 1137 prevalent cases of diabetes. Cross-sectional multivariableadjusted analyses showed the prevalence odds ratio of having baseline diabetes was 0.79 (95\% CI: $0.67,0.94)$ for those with any history of migraine. We further stratified our results by migraine subtype and found the prevalence odds of having diabetes was 0.78 (95\% CI: $0.57,1.07$ ) for women with active migraine with aura, 0.70 (95\% CI: $0.54,0.91)$ for women with active migraine without aura, and $0.92(95 \% \mathrm{CI}: 0.71,1.19)$ for women with a past history of migraine compared to women with no history of migraine.

After excluding the women who reported diabetes at baseline, we were left with 38,620 women for our incident diabetes analysis. Of these 38,620 women, $5062(13.1 \%)$ had active migraine, of whom 2014 (39.8\%) reported migraine with aura, and 2,087 (5.4\%) had a past history of migraine. The baseline characteristics of the women who did not have baseline diabetes at baseline can be seen in Table 1. Compared with women without a history of migraine, women who experienced migraine were younger and more likely to be currently taking NSAIDs more than one day per week.

During a mean of 14.6 years of follow-up (564,508 person years) there were 3,032 cases of confirmed incident T2D. Any history of migraine was not associated with a significantly increased risk of T2D (HR=1.06, 95\% CI: 0.97, 1.16) in multivariable analysis. We then further stratified our results by migraine subtype. Age- and multivariable-adjusted analysis 
did not show a statistically significant increase in the risk of diabetes for active migraine with or without aura compared to those without a history of migraine (Table 2). While those with a past history of migraine had a significant increase in their risk of T2D in an ageadjusted model (HR=1.29, 95\% CI: 1.12-1.49), this association was no longer significant after adjusting for other potential confounders (HR=1.13, 95\% CI: 0.98, 1.30) (Table 2).

We did not find evidence of effect modification by randomized treatment assignment to aspirin, currently taking NSAIDs more than 1 day per week, history of hypertension, baseline treatment with medication for high blood pressure, obesity, or age (all p-values $>0.12$ ).

We did not find evidence of an increased risk of T2D with increasing frequency of active migraine. Compared to those active migraineurs who experience migraines less than six times per year, active migraineurs who experience migraines every other month, monthly and weekly have hazard ratio (95\% CIs) for diabetes of $1.24(0.92,1.68), 0.85(0.65,1.12)$ and $1.21(0.80,1.84)$ respectively.

\section{Discussion}

Results from this large prospective cohort of women do not suggest an association between migraine and incident T2D. Compared with women without migraine, women who report a past history of migraine, active migraine with aura or active migraine without aura were not at increased risk of developing T2D. We further had no evidence that the lack of association was modified by age, randomized treatment assignment to aspirin, currently taking NSAIDs more than 1 day per week, history of hypertension, baseline treatment with medication for high blood pressure, or obesity.

\section{Comparison with other studies}

Only a few cross-sectional studies have evaluated the relationship between migraine status and prevalent diabetes in population-based samples. One study examined the association between migraine and cardiovascular risk factors including diabetes in 1450 elderly Brazilians (average age $=71.9$ years) (24). Assessment of diabetes was validated by fasting blood glucose or use of oral hypoglycemics or insulin and migraine was assessed according to ICHD-II criteria. In a multivariable logistic regression model, there was no statistically significant association between diabetes and migraine (odds ratio $[\mathrm{OR}]=1.19 ; 95 \%$ CI: 0.81 , 1.76). The study did not collect information about aura thus limiting analysis by migraine subtype. Another cross-sectional study among Danish twins which did stratify by migraine subtype found no association between any migraine, migraine with aura, or migraine without aura and diabetes (25).

Data from the large cross-sectional population-based American Migraine Prevalence and Prevention study (AMPP) showed a moderate increase in the likelihood of a diagnosis of diabetes in subjects who reported having a migraine in the last 12 months compared with headache-free controls ( $12.6 \%$ vs $9.4 \%$; OR=1.4, 95\% CI: $1.2-1.4)$. Similar results were obtained for subjects with migraine with aura and migraine without aura overall $(3,26)$. In the same population, the prevalence of diabetes did not differ between subjects with episodic and chronic migraine (27). When the results were stratified by gender, women who experience any migraine had an increased risk of diabetes (OR=1.28, 95\% CI: 1.10, 1.49) which was driven by the migraine with aura group ( $\mathrm{OR}=1.47,95 \% \mathrm{CI}: 1.22,1.78)$.

Our study, in contrast to the findings of the AMPP, showed a lower prevalence of diabetes among women with any history of migraine. Analysis stratified by migraine subtype showed that this inverse association was driven by the migraine without aura subgroup. One 
potential explanation for the different results between WHS and AMPP may be the different age distributions of the AMPP study and the WHS. The AMPP study had a median age of 20.4 among headache-free controls and 24.0 among migraineurs. In contrast, all participants in the WHS were aged 45 or older. Results of the AMPP study among men and women combined showed that the prevalence of diabetes differed by age and was highest amongst people aged 18-29 with migraine with aura (OR=2.65, 95\% CI: 0.87-8.09) (3). The hypothesis that the association between migraine and diabetes is stronger among young age groups was also suggested by a longitudinal cohort study of Finnish children which showed that at age 15, diabetes was found more often in those with migraine than those without (28). These results may suggest that migraine more strongly associates with Type 1 diabetes (T1D), which is more common among younger individuals and is in contrast to T2D results primarily from autoimmune destruction of the pancreas. This could add to the evidence that migraine may associate with auto-immune conditions (29).

While most studies did not distinguish between type 1 and type 2 diabetes, the Head-HUNT study, a large cross-sectional population-based study in Norway, found that subjects with diagnosed Type 1 and Type 2 diabetes were less likely to report having suffered headache or migraine in the previous year than those without diabetes in cross-sectional analyses (multivariable adjusted ORs were $0.4,95 \%$ CI: $0.2-0.9$ for T1D and migraine and $0.7,95 \%$ CI: $0.5-0.9$ for T2D and migraine). The inverse association observed in this study between migraine and T2D diabetes is similar to that seen in the cross-sectional analysis from our study. In that study, a decreased odds of reporting migraine was associated with duration of diabetes and $\mathrm{HbA} 1 \mathrm{c}>6.6 \%$, which the authors suggested might be due to diabetic neuropathy, among other possibilities (30). Since the subjects in the Head-HUNT study are older than those in the AMPP study and the Finnish study, the inverse association between migraine and T1D in this study may relate to the relatively longer duration of disease.

\section{Strengths and Limitations}

Our study has a number of important strengths. It is a large, prospective study with validation of exposure (migraine status) and outcome (diabetes), a long follow-up period and a large number of outcome events. We were able to adjust for a large number of potential confounders, including age, sex, BMI, exercise, and alcohol.

Our study does, however, have some limitations which should be considered when interpreting our results. Although validation studies of migraine status have shown good agreement between self-reported migraine status and ICHD criteria $(4,21)$, migraine status may still have been misclassified. Since we would not expect the misclassification of migraine status to vary between patients who developed diabetes and those who did not, this misclassification is most likely random. Validation studies have also shown that women who report having diabetes are likely to truly have the disease $(31,32)$, but it is possible that some women may not have reported diabetes diagnoses and we may have missed some diabetes cases as diabetes can be asymptomatic at initial onset. However, we would not expect differences in reporting diabetes diagnoses among our migraine groups. Since this population is primarily composed of white females, health professionals, 45 years of age or older it is possible that our results may not be generalizable to other populations. Lastly, although we adjusted for a large number of potential confounders, residual or unmeasured confounding remains a possibility as our study is observational.

In summary, this longitudinal cohort study of middle-aged and older female health professionals did not find an association between migraine subtypes and incident diabetes. Our results do not support the hypothesis that migraineurs are at increased risk for T2D. A possible inverse association between T2D and active migraine may deserve further 
evaluation. Future research regarding the influence of diabetes on the clinical course of migraine may also be warranted.

\section{Acknowledgments}

Funding

The WHS is supported by grants from the National Institutes of Health (HL-043851, HL-080467, HL-099355, and CA-047988). Pamela Rist is funded by a training grant from the National Institute of Aging (AG-00158). Rebecca Burch is supported by a fellowship from the American Headache Society.

\section{References}

1. Bigal ME, Lipton RB. The epidemiology, burden, and comorbidities of migraine. Neurol Clin. 2009; 27:321-34. [PubMed: 19289218]

2. Goadsby PJ, Lipton RB, Ferrari MD. Migraine--current understanding and treatment. N Engl J Med. 2002; 346:257-70. [PubMed: 11807151]

3. Bigal ME, Kurth T, Santanello N, Buse D, Golden W, Robbins M, Lipton RB. Migraine and cardiovascular disease: a population-based study. Neurology. 2010; 74:628-35. [PubMed: 20147658]

4. Kurth T, Gaziano JM, Cook NR, Logroscino G, Diener HC, Buring JE. Migraine and risk of cardiovascular disease in women. Jama. 2006; 296:283-91. [PubMed: 16849661]

5. Kurth T, Kase CS, Schurks M, Tzourio C, Buring JE. Migraine and risk of haemorrhagic stroke in women: prospective cohort study. Bmj. 2010; 341:c3659. [PubMed: 20736268]

6. Kurth T, Slomke MA, Kase CS, Cook NR, Lee IM, Gaziano JM, et al. Migraine, headache, and the risk of stroke in women: a prospective study. Neurology. 2005; 64:1020-6. [PubMed: 15781820]

7. MacClellan LR, Giles W, Cole J, Wozniak M, Stern B, Mitchell BD, Kittner SJ. Probable migraine with visual aura and risk of ischemic stroke: the stroke prevention in young women study. Stroke. 2007; 38:2438-45. [PubMed: 17690308]

8. Schurks M, Rist PM, Bigal ME, Buring JE, Lipton RB, Kurth T. Migraine and cardiovascular disease: systematic review and meta-analysis. Bmj. 2009; 339:b3914. [PubMed: 19861375]

9. Hamed SA, Hamed EA, Ezz Eldin AM, Mahmoud NM. Vascular risk factors, endothelial function, and carotid thickness in patients with migraine: relationship to atherosclerosis. J Stroke Cerebrovasc Dis. 2010; 19:92-103. [PubMed: 20189084]

10. Scher AI, Terwindt GM, Picavet HS, Verschuren WM, Ferrari MD, Launer LJ. Cardiovascular risk factors and migraine: the GEM population-based study. Neurology. 2005; 64:614-20. [PubMed: 15728281]

11. Kurth T, Ridker PM, Buring JE. Migraine and biomarkers of cardiovascular disease in women. Cephalalgia. 2008; 28:49-56. [PubMed: 17986270]

12. Tietjen GE, Herial NA, White L, Utley C, Kosmyna JM, Khuder SA. Migraine and biomarkers of endothelial activation in young women. Stroke. 2009; 40:2977-82. [PubMed: 19608996]

13. Bigal ME, Kurth T, Hu H, Santanello N, Lipton RB. Migraine and cardiovascular disease: possible mechanisms of interaction. Neurology. 2009; 72:1864-71. [PubMed: 19470970]

14. Peterlin BL, Rapoport AM, Kurth T. Migraine and obesity: epidemiology, mechanisms, and implications. Headache. 2010; 50:631-48. [PubMed: 19845784]

15. Cavestro C, Rosatello A, Micca G, Ravotto M, Marino MP, Asteggiano G, Beghi E. Insulin metabolism is altered in migraineurs: a new pathogenic mechanism for migraine? Headache. 2007; 47:1436-42. [PubMed: 18052953]

16. Guldiken B, Guldiken S, Demir M, Turgut N, Kabayel L, Ozkan H, et al. Insulin resistance and high sensitivity C-reactive protein in migraine. Can J Neurol Sci. 2008; 35:448-51. [PubMed: 18973061]

17. Rainero I, Limone P, Ferrero M, Valfre W, Pelissetto C, Rubino E, et al. Insulin sensitivity is impaired in patients with migraine. Cephalalgia. 2005; 25:593-7. [PubMed: 16033384] 
18. Rexrode KM, Lee IM, Cook NR, Hennekens CH, Buring JE. Baseline characteristics of participants in the Women's Health Study. J Womens Health Gend Based Med. 2000; 9:19-27. [PubMed: 10718501]

19. Lee IM, Cook NR, Gaziano JM, Gordon D, Ridker PM, Manson JE, et al. Vitamin E in the primary prevention of cardiovascular disease and cancer: the Women's Health Study: a randomized controlled trial. JAMA. 2005; 294:56-65. [PubMed: 15998891]

20. Ridker PM, Cook NR, Lee IM, Gordon D, Gaziano JM, Manson JE, et al. A randomized trial of low-dose aspirin in the primary prevention of cardiovascular disease in women. N Engl J Med. 2005; 352:1293-304. [PubMed: 15753114]

21. Schurks M, Buring JE, Kurth T. Agreement of self-reported migraine with ICHD-II criteria in the Women's Health Study. Cephalalgia. 2009; 29:1086-90. [PubMed: 19735535]

22. Report of the Expert Committee on the Diagnosis and Classification of Diabetes Mellitus. Diabetes Care. 1997; 20:1183-97. [PubMed: 9203460]

23. Ding EL, Song Y, Manson JE, Pradhan AD, Buring JE, Liu S. Accuracy of administrative coding for type 2 diabetes in children, adolescents, and young adults. Diabetes Care. 2007; 30:e98. author reply e9. [PubMed: 17726188]

24. Bensenor IM, Goulart AC, Lotufo PA, Menezes PR, Scazufca M. Cardiovascular risk factors associated with migraine among the elderly with a low income: the Sao Paulo Ageing \& Health Study (SPAH). Cephalalgia. 2011; 31:331-7. [PubMed: 20693229]

25. Le H, Tfelt-Hansen P, Russell MB, Skytthe A, Kyvik KO, Olesen J. Co-morbidity of migraine with somatic disease in a large population-based study. Cephalalgia. 31:43-64. [PubMed: 20974590]

26. Bigal ME, Golden W, Buse D, Chen YT, Lipton RB. Triptan use as a function of cardiovascular risk. A population-based study. Headache. 2010; 50:256-63. [PubMed: 20039953]

27. Buse DC, Manack A, Serrano D, Turkel C, Lipton RB. Sociodemographic and comorbidity profiles of chronic migraine and episodic migraine sufferers. J Neurol Neurosurg Psychiatry. 2010; 81:428-32. [PubMed: 20164501]

28. Sillanpaa M, Aro H. Headache in teenagers: comorbidity and prognosis. Funct Neurol. 2000; 15 (Suppl 3):116-21. [PubMed: 11200781]

29. Kister I, Munger KL, Herbert J, Ascherio A. Increased risk of multiple sclerosis among women with migraine in the Nurses' Health Study II. Mult Scler. 2012; 18:90-7. [PubMed: 21816759]

30. Aamodt AH, Stovner LJ, Midthjell K, Hagen K, Zwart JA. Headache prevalence related to diabetes mellitus. The Head-HUNT study. Eur J Neurol. 2007; 14:738-44. [PubMed: 17594328]

31. Pradhan AD, Manson JE, Rifai N, Buring JE, Ridker PM. C-reactive protein, interleukin 6, and risk of developing type 2 diabetes mellitus. Jama. 2001; 286:327-34. [PubMed: 11466099]

32. Song Y, Manson JE, Buring JE, Liu S. A prospective study of red meat consumption and type 2 diabetes in middle-aged and elderly women: the women's health study. Diabetes Care. 2004; 27:2108-15. [PubMed: 15333470] 
Table 1

Baseline characteristics of women without diabetes at baseline according to migraine status in the Women's Health Study $(\mathrm{n}=38,620)$.

\begin{tabular}{|c|c|c|c|c|}
\hline \multirow[b]{2}{*}{ Characteristic } & \multirow[b]{2}{*}{$\begin{array}{l}\text { No migraine history, } \\
\mathrm{n}=\mathbf{3 1}, \mathbf{4 7 1}\end{array}$} & \multicolumn{2}{|l|}{ Migraine } & \multirow[b]{2}{*}{$\begin{array}{l}\text { History of migraine, } \\
\mathbf{n}=\mathbf{2 , 0 8 7}\end{array}$} \\
\hline & & $\begin{array}{l}\text { Migraine with } \\
\text { aura, } n=2,014\end{array}$ & $\begin{array}{l}\text { Migraine without } \\
\text { aura, } n=3,048\end{array}$ & \\
\hline Mean (SE) age, y & $54.8(7.1)$ & $52.9(6.1)$ & $52.6(5.6)$ & $55.5(7.4)$ \\
\hline Body mass index (SE), $\mathrm{kg} / \mathrm{m}^{2}$ & $25.9(5.0)$ & $25.9(4.8)$ & $26.1(5.0)$ & $26.1(5.1)$ \\
\hline History of hypertension, $\%$ & 24.4 & 24.3 & 24.0 & 32.2 \\
\hline Antihypertensive medication use, $\%$ & 12.6 & 12.2 & 13.2 & 18.3 \\
\hline History of high cholesterol, $\%$ & 28.4 & 31.3 & 30.2 & 34.0 \\
\hline Cholesterol lowering medication use, $\%$ & 3.0 & 2.6 & 2.7 & 3.3 \\
\hline Postmenopausal, \% & 55.0 & 46.6 & 46.9 & 58.5 \\
\hline $\begin{array}{l}\text { Ever used oral contraceptive for } \geq 2 \text { months, } \\
\%\end{array}$ & 68.5 & 76.6 & 75.2 & 70.2 \\
\hline \multicolumn{5}{|l|}{ Postmenopausal hormone use, $\%$} \\
\hline Never & 48.6 & 41.7 & 44.9 & 43.8 \\
\hline Past & 10.0 & 9.8 & 9.2 & 12.8 \\
\hline Current & 41.2 & 48.3 & 45.7 & 43.2 \\
\hline \multicolumn{5}{|l|}{ Alcohol consumption, $\%$} \\
\hline Rarely/never & 43.7 & 48.3 & 48.3 & 44.3 \\
\hline 1-3 drinks/month & 12.9 & 13.7 & 14.9 & 14.5 \\
\hline 1-6 drinks/week & 32.5 & 30.7 & 29.8 & 30.4 \\
\hline$\geq 1$ drink per day & 10.9 & 7.4 & 7.0 & 10.8 \\
\hline Randomized aspirin assignment, $\%$ & 50.0 & 49.8 & 50.0 & 48.2 \\
\hline Randomized vitamin $\mathrm{E}$ assignment, \% & 50.1 & 51.0 & 48.4 & 50.3 \\
\hline \multicolumn{5}{|l|}{ Vigorous physical activity, $\%$} \\
\hline Rarely/never & 37.9 & 37.4 & 38.3 & 39.1 \\
\hline$<1 /$ week & 19.4 & 22.4 & 23.1 & 20.9 \\
\hline 1-3 times/week & 31.7 & 30.7 & 29.6 & 29.3 \\
\hline$\geq 4$ times/week & 11.0 & 9.5 & 9.0 & 10.5 \\
\hline \multicolumn{5}{|l|}{ Smoking status, $\%$} \\
\hline Never & 50.5 & 53.8 & 55.3 & 50.1 \\
\hline Past & 36.2 & 33.7 & 33.9 & 34.6 \\
\hline Current $<15$ cig/day & 4.9 & 4.9 & 3.5 & 5.8 \\
\hline Current $\geq 15$ cig/day & 8.2 & 7.3 & 7.1 & 9.4 \\
\hline $\begin{array}{l}\text { Currently taking NSAIDs more than } 1 \text { day/ } \\
\text { week }\end{array}$ & 9.7 & 15.4 & 15.9 & 14.3 \\
\hline Family history of diabetes, $\%$ & 24.7 & 23.8 & 25.3 & 26.8 \\
\hline
\end{tabular}

Numbers may not add up to $100 \%$ because of rounding or missing data. 
Table 2

Age- and multivariable-adjusted hazard ratios for incident diabetes according to migraine status $(\mathrm{N}=38,620)$.

\begin{tabular}{lccccc} 
& \multicolumn{2}{c}{ Age-adjusted } & & \multicolumn{2}{c}{ Multivariable-adjusted $^{*}$} \\
\cline { 2 - 3 } \cline { 5 - 6 } Migraine status & No. of cases & Hazard ratios $(\mathbf{9 5 \%} \mathbf{C I})$ & & No. of cases & Hazard ratios $(\mathbf{9 5 \%}$ CI) \\
\hline No history & 2406 & 1.00 & 2406 & 1.00 \\
MA & 167 & $1.10(0.94,1.29)$ & & 167 & $1.06(0.91,1.24)$ \\
MO & 256 & $1.11(0.97,1.26)$ & & 256 & $1.01(0.89,1.16)$ \\
Past history & 203 & $1.29(1.12,1.49)$ & & 203 & $1.13(0.98,1.30)$ \\
\hline
\end{tabular}

CI indicates confidence interval

Adjusted for age, body mass index, exercise, ever used postmenopausal hormones of any type, postmenopausal status, randomized treatment assignment to aspirin and vitamin $\mathrm{E}$, alcohol consumption, history of hypertension $\geq 140 / 90 \mathrm{mmHg}$, baseline treatment with medication for high blood pressure, history of high cholesterol $\geq 240 \mathrm{mg} / \mathrm{dL}$, baseline treatment with cholesterol-lowering medication, smoking status, systolic blood pressure, family history of diabetes, and ever used oral contraceptives $\geq 2$ months. 